\title{
System size stochastic resonance: General nonequilibrium potential framework
}

\author{
B. von Haeften, ${ }^{1}$ G. Izús, ${ }^{1,2, *}$ and H. S. Wio ${ }^{3,4, \dagger}$ \\ ${ }^{1}$ Departamento de Física, FCEyN, Universidad Nacional de Mar del Plata, Deán Funes 3350, 7600 Mar del Plata, Argentine \\ ${ }^{2}$ IMEDEA (CSIC-UIB), E-07122 Palma de Mallorca, Spain \\ ${ }^{3}$ Instituto de Física de Cantabria, Universidad de Cantabria, E-39005 Santander, Spain \\ ${ }^{4}$ Centro Atómico Bariloche and Instituto Balseiro, 8400 San Carlos de Bariloche, Argentine
}

(Received 9 March 2005; published 1 August 2005)

\begin{abstract}
We study the phenomenon of system size stochastic resonance within the nonequilibrium potential framework. We analyze three different cases of spatially extended systems, exploiting the knowledge of their nonequilibrium potential, showing that through the analysis of that potential we can obtain a clear physical interpretation of this phenomenon in wide classes of extended systems. Depending on the characteristics of the system, the phenomenon is associated with a breaking of the symmetry of the nonequilibrium potential or a deepening of the potential minima yielding an effective scaling of the noise intensity with the system size.
\end{abstract}

DOI: 10.1103/PhysRevE.72.021101

PACS number(s): 05.40.-a, 02.50.Ey, 87.10.+e

\section{INTRODUCTION}

The phenomenon of stochastic resonance (SR)—namely, the enhancement of the output signal-to-noise ratio (SNR) caused by injection of an optimal amount of noise into a nonlinear system-configures a counterintuitive cooperative effect arising from the interplay between deterministic and random dynamics in a nonlinear system. The broad range of phenomena for which this mechanism can offer an explanation can be appreciated in Refs. $[1,2]$ and references therein, where we can scan the state of the art.

Most of the phenomena that could possibly be described within a SR framework occur in extended systems: for example, diverse experiments were carried out to explore the role of SR in sensory and other biological functions [3] or in chemical systems [4]. These were, together with the possible technological applications, the main motivation for many recent studies showing the possibility of achieving an enhancement of the system response by means of the coupling of several units into what conforms to an extended medium [5-10], or analyzing the possibility of making the system response less dependent on a fine tuning of the noise intensity, as well as different ways to control the phenomenon $[11,12]$.

In previous papers $[7-10,13]$ we have studied the stochastic resonant phenomenon in extended systems for the transition between two different patterns, exploiting the concept of the nonequilibrium potential (NEP) [14,15]. This potential is a special Lyapunov functional of the associated deterministic system which for nonequilibrium systems plays a role similar to that played by a thermodynamic potential in equilibrium thermodynamics [14]. Such a nonequilibrium potential, closely related to the solution of the time independent Fokker-Planck equation of the system, characterizes the global properties of the dynamics, that is, the attractors, the relative (or nonlinear) stability of these attractors, the height of the barriers separating attraction basins, and in addition it

\footnotetext{
*Electronic address: izus@mdp.edu.ar

†Electronic address: wio@ifca.unican.es
}

allows us to evaluate the transition rates among the different attractors $[14,15]$. In another recent paper we have explored the characteristics of this SR phenomenon in an extended system composed of an ensemble of noise-induced nonlinear oscillators coupled by a nonhomogeneous, density dependent diffusion, externally forced and perturbed by a multiplicative noise, that shows an effective noise induced bistable dynamics [16]. The stochastic resonance between the attractors of the noise-induced dynamics was theoretically investigated in terms of a two-state approximation. It was shown that the knowledge of the exact NEP allowed us to completely analyze the behavior of the output SNR.

Recent studies on biological models of the HodgkinHuxley type $[17,18]$ have shown that ion concentrations along biological cell membranes present intrinsic SR-like phenomena as the number of ion channels is varied. A related result [19] shows that even in the absence of external forcing, the regularity of the collective firing of a set of coupled excitable FitzHugh-Nagumo units becomes optimal for a given value of the number of elements. From a physical system point of view, the same phenomenon-which has been called system size stochastic resonance (SSSR) - has also been found in an Ising model as well as in a set of globally coupled units described by a $\phi^{4}$ theory [20]. It was even shown to arise in opinion formation models [21].

The SSSR phenomenon occurs in extended systems; hence it is clearly of great interest to describe this phenomenon within the NEP framework. More, the NEP offers a general framework for the study of the dependence of resonant and other related phenomena on any of the system's parameters. With such a goal in mind, in a recent work [22] it was shown that SSSR could be analyzed within a NEP framework and that, depending on the system, its origin could be essentially traced back to a breaking of the symmetry of such a potential. Even those cases discussed in [20] could be described within this same framework, and the ("effective") scaling of the noise with the system's size could be clearly seen. Here, we discuss in more detail the cases analyzed in $[20,22]$ and present another interesting one, corresponding to the study of SSSR in a system that also shows noise-induced patterns, the same one studied in [16]. We 
show that in two of the cases (corresponding to pattern forming systems that include only local interactions), the problem can be rewritten in such a way as to present a kind of "entrainment" between the symmetry breaking of an "effective" potential together with a scaling of the noise intensity with the system size.

The organization of the paper is as follows. In Sec. II we focus on a simple reaction-diffusion model with a known form of the NEP, which presents SSSR associated with a NEP's symmetry breaking. In Sec. III we analyze the model of globally coupled nonlinear oscillators discussed in [20], and show that it can also be described within the NEP framework, with SSSR arising due to a deepening of the potential wells, or through an effective scaling of the noise intensity with the system size. In Sec. IV we briefly review the model to be used for the case of multiplicative noise and analyze its behavior. Finally, we present in Sec. V some conclusions and perspectives.

\section{A SIMPLE REACTION-DIFFUSION SYSTEM}

\section{Brief review of the model}

The specific model we shall focus on in this section, with a known form of the NEP, corresponds to a one-dimensional, one-component model $[23,24]$ that, with a piecewise linear form for the reaction term, mimics general bistable reactiondiffusion models [23], that is, with a cubiclike nonlinear reaction term. In particular we will exploit some of the results on the influence of general boundary conditions (called albedo) found in [25] as well as previous studies of the NEP [15] and of SR [7-10].

The particular nondimensional form of the model that we work with is $[7,8,25]$

$$
\frac{\partial}{\partial t} \phi=D \frac{\partial^{2}}{\partial y^{2}} \phi-\phi+\phi_{h} \theta\left(\phi-\phi_{c}\right) \text {. }
$$

Here $\theta(z)$ is the Heaviside step function, and $\phi_{c}$ is the value at which the piecewise linear "nonlinearity" has a jump. We consider here a class of stationary structures $\phi(y)$ in the bounded domain $y \in\left[-y_{L}, y_{L}\right]$ with albedo boundary conditions at both ends,

$$
\left.\frac{\partial \phi(y, t)}{\partial y}\right|_{y= \pm y_{L}}=\mp k \phi\left( \pm y_{L}, t\right),
$$

where $k>0$ is the albedo parameter. It is worth noting that for $k \rightarrow 0$ we recover the usual case of Neumann boundary conditions (i.e., $\left.[\partial \phi(y, t) / \partial y]\right|_{y= \pm y_{L}}=0$ ), while for $k \rightarrow \infty$ what results are the usual Dirichlet boundary conditions $\left[\phi\left(y= \pm y_{L}, t\right)=0\right]$.

Those stationary structures are the spatially symmetric (stable) solutions to Eq. (1) already studied in [25]. The explicit form of these stationary patterns is shown in Eq. (9) of [25], while the double-valued coordinate $y_{c}^{ \pm}$, at which $\phi$ $=\phi_{c}$, is given by Eq. (11) also in [25]. In order to have a bistable behavior we require $0<\phi_{c}<\phi_{h}$. Typical forms of the patterns are shown in Fig. 1.

When $y_{c}^{ \pm}$exists and $y_{c}^{ \pm}<y_{L}$, this pair of solutions represents a structure with a central "excited" zone $\left(\phi>\phi_{c}\right)$ and

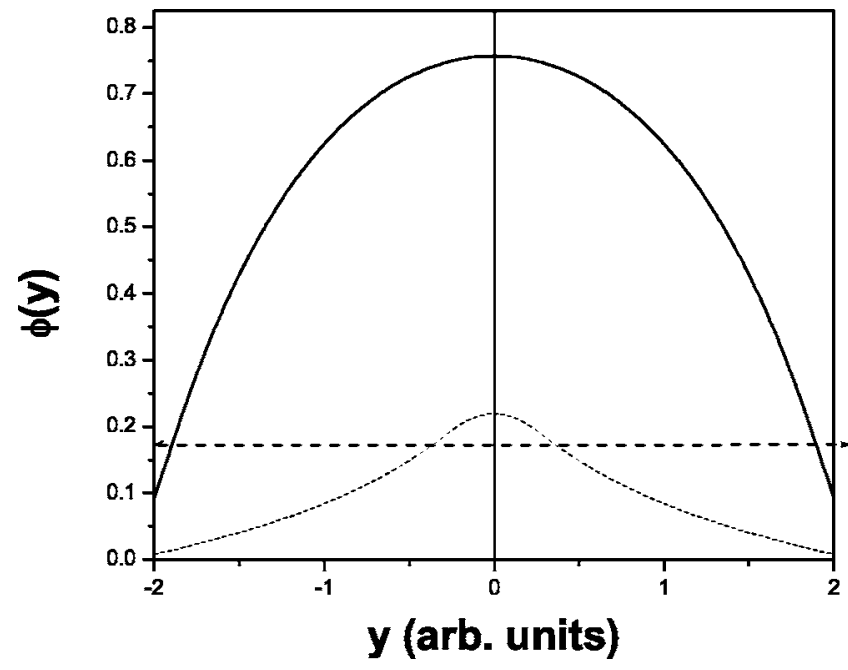

FIG. 1. Typical form of the patterns $\phi(y)$, for $D=1, y_{L}=2, k$ $=2$, and $\phi_{c} / \phi_{h}=0.193$. The continuous line corresponds to $\phi_{1}$, the stable nonhomogeneous pattern; the dotted line is $\phi_{u}$, the unstable pattern. In addition we also have the always present $\phi_{0}$, the stable null pattern that coincides with the horizontal axis. The dashed line indicates the value of $\phi_{c}$.

two lateral "resting" regions $\left(\phi<\phi_{c}\right)$. For each parameter set, there are two stationary solutions, given by the two values of $y_{c}$. Figure 5 in [25], which we do not reproduce here, depicts the curves corresponding to the relation $y_{c} / y_{L}$ vs $k$, for various values of $\phi_{c} / \phi_{h}$.

Through a linear stability analysis it has been shown [25] that the structure with the smallest excited region [that is, with $y_{c}=y_{c}^{+}$, denoted by $\left.\phi_{u}(y)\right]$ is unstable, whereas the other one [with $y_{c}=y_{c}^{-}$, denoted by $\left.\phi_{1}(y)\right]$ is linearly stable. The trivial homogeneous solution $\phi_{0}(y)=0$ (denoted by $\phi_{0}$ ) exists for any parameter set and is always linearly stable. These two linearly stable solutions $\left(\phi_{0}\right.$ and $\left.\phi_{1}\right)$ are the only stable stationary structures under the given albedo boundary conditions. We will concentrate on the region of values of $\phi_{c} / \phi_{h}, y_{L}$, and $k$, where both nonhomogeneous structures exist.

For the system with the albedo boundary conditions (BCs) that we are considering here, the NEP reads [15]

$$
\begin{aligned}
\mathcal{F}\left[\phi, k, y_{L}\right]= & \int_{-y_{L}}^{y_{L}}\left\{-\int_{0}^{\phi(y, t)}\left[-\phi^{\prime}+\phi_{h} \theta\left(\phi^{\prime}-\phi_{c}\right)\right] d \phi^{\prime}\right. \\
& \left.+\frac{D}{2}\left(\frac{\partial}{\partial y} \phi(y, t)\right)^{2}\right\} d y+\left.\frac{k}{2} \phi(y, t)^{2}\right|_{ \pm y_{L}} .
\end{aligned}
$$

Strictly speaking, this is the system's Lyapunov functional, as we are still considering the deterministic case. However, in what follows we will always refer to the NEP for both the deterministic and stochastic cases. This functional satisfies the "potential" condition $(\partial / \partial t) \phi(y, t)$ $=-[\delta / \delta \phi(y, t)] \mathcal{F}\left[\phi, k, y_{L}\right]$, where $\delta / \delta \phi(y, t)$ indicates a functional derivative. 


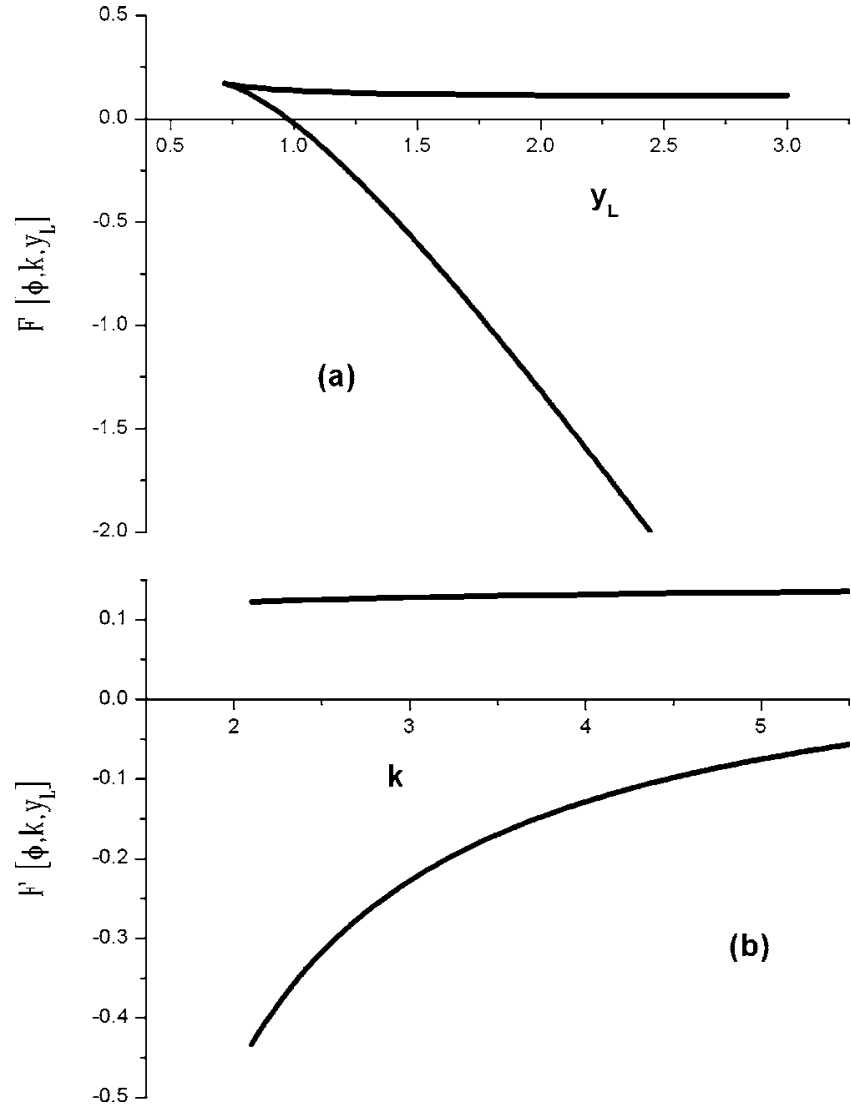

FIG. 2. NEP evaluated at the stationary solutions $\phi_{0}(y), \phi_{1}(y)$, and $\phi_{u}(y)$ for $\mathcal{F}[\phi, k, y L]$ vs (a) $y_{L}$ and (b) $k$. In the first case $k$ $=3$, and $y_{L}=1.2$ in the second. In both cases we have fixed $D=1$, $\phi_{c} / \phi_{h}=0.193$, and the NEP for $\phi_{0}(y)$ coincides with the horizontal axis.

Replacing the explicit forms of the stationary nonhomogeneous solutions [Eq. (9) in [25]], we obtain the explicit expression $[7,15]$

$$
\begin{aligned}
\mathcal{F}^{u, 1}= & \mathcal{F}\left[\phi_{u, 1}, k, y_{L}\right]=-\phi_{h}^{2} y_{c}^{ \pm}\left(1-2 \frac{\phi_{c}}{\phi_{h}}\right) \\
& +\phi_{h}^{2} \sinh \left(y_{c}^{ \pm} / \sqrt{D}\right) \frac{\rho\left(k,\left(y_{L}-y_{c}^{ \pm}\right) / \sqrt{D}\right)}{\rho\left(k, y_{L} / \sqrt{D}\right)}
\end{aligned}
$$

here $\rho(k, \zeta)=\sinh (\zeta)+k \cosh (\zeta)$, and $\rho^{\prime}(k, \zeta)=\partial \rho(k, \zeta) / \partial \zeta$, while for the homogeneous trivial solution $\phi_{0}=0$, we have instead $\mathcal{F}\left[\phi_{0}, k, y_{L}\right]=\mathcal{F}^{0}=0$.

Figure 2(a) depicts the nonequilibrium potential $\mathcal{F}\left[\phi, k, y_{L}\right]$ as a function of the system size $y_{L}$, for a fixed albedo parameter $k$, and a fixed value of $\phi_{c} / \phi_{h}$. The curves correspond to the NEP evaluated on the nonhomogeneous structures $\mathcal{F}^{u, 1}$, whereas $\mathcal{F}^{0}$, the NEP of the trivial solution, is a line coincident with the horizontal axis. We have focused on the bistable zone, the upper branch being the NEP of the unstable structure, where $\mathcal{F}$ attains a maximum, while in the lower branch (for $\phi=\phi_{0}$ or $\phi=\phi_{1}$ ), the NEP has local minima. We see that when $y_{L}$ becomes small, the difference between the NEP for the states $\phi_{u}(y)$ and $\phi_{1}(y)$ is reduced until, for $y_{L} \approx 0.72$, they coalesce and, for even lower values of $y_{L}$, disappear (inverse saddle-node bifurcation).

It is important to note that, since the NEP for the unstable solution $\phi_{u}$ is always positive and, for the stable nonhomogeneous structure $\phi_{1}, \mathcal{F}^{1}<0$ for $y_{L}$ large enough, and $\mathcal{F}^{1}$ $>0$ for small values of $y_{L}$, the NEP for this structure vanishes for an intermediate value $y_{L}=y_{L}^{*} \approx 1$ of the system size. At that point, the stable nonhomogeneous structure $\phi_{1}(y)$ and the trivial solution $\phi_{0}(y)$ exchange their relative stability.

For completeness and later use, in Fig. 2(b) we show $\mathcal{F}\left[\phi, k, y_{L}\right]$ but now as a function of $k$, for a fixed value of $y_{L}$ and the same value of $\phi_{c} / \phi_{h}$. In this case we see that there is no crossing between the curves corresponding to the states $\phi_{1}(y)$ and $\phi_{0}(y)$, and correspondingly no stability exchange. Also, the initial large difference between the NEP for the states $\phi_{u}(y)$ and $\phi_{1}(y)$ is reduced for increasing $k$ until, for $k \rightarrow \infty$, the values of $\mathcal{F}\left[\phi, k, y_{L}\right]$ for Dirichlet (BCs) are asymptotically reached.

\section{B. System size stochastic resonance}

In order to account for the effect of fluctuations, we include in the time-evolution equation of our model [Eq. (1)] a fluctuation term, which we model as an additive noise source $[10,26]$, yielding a stochastic partial differential equation for the random field $\phi(y, t)$,

$$
\frac{\partial}{\partial t} \phi(y, t)=D \frac{\partial^{2}}{\partial y^{2}} \phi-\phi+\phi_{h} \theta\left(\phi-\phi_{c}\right)+\xi(y, t) .
$$

We make the simplest assumptions about the fluctuation term $\xi(y, t)$, i.e., that it is a Gaussian white noise with zero mean and a correlation function given by $\left\langle\xi(y, t) \xi\left(y^{\prime}, t^{\prime}\right)\right\rangle=2 \gamma \delta(t$ $\left.-t^{\prime}\right) \delta\left(y-y^{\prime}\right)$, where $\gamma$ denotes the noise strength.

As was discussed in [7-10], using known results for activation processes in multidimensional systems [27], we can estimate the activation rate according to the following Kramers'-like result for $\langle\tau\rangle$, the mean first-passage time for the transitions between attractors,

$$
\left\langle\tau_{i}\right\rangle=\tau_{0} \exp \left\{\frac{\Delta \mathcal{F}^{i}\left[\phi, k, y_{L}\right]}{\gamma}\right\},
$$

where

$$
\Delta \mathcal{F}\left[\phi, k, y_{L}\right]=\mathcal{F}\left[\phi_{u}(y), k, y_{L}\right]-\mathcal{F}\left[\phi_{i}(y), k, y_{L}\right]
$$

$(i=0,1)$. The prefactor $\tau_{0}$ is usually determined by the curvature of $\mathcal{F}\left[\phi, k, y_{L}\right]$ at its extremes and typically is, on one hand, several orders of magnitude smaller than the average time $\langle\tau\rangle$, while on the other hand (around the bistable point) it does not change significantly when varying the system's parameters. Hence, in order to simplify the analysis, we assume here that $\tau_{0}$ is constant and scale it out of our results. The behavior of $\langle\tau\rangle$ as a function of the different parameters $\left(k, \phi_{c} / \phi_{h}\right)$ was shown in $[7,8,15]$.

As was done in [7], we assume now that the system is (adiabatically) subject to an external harmonic variation of the parameter $\phi_{c}, \phi_{c}(t)=\phi_{c}^{*}+\delta \phi_{c} \cos (\omega t)[8,10]$, and exploit the two-state approximation [1] as in [8-10]. Such an approximation basically consist in reducing the whole dynam- 
ics on the bistable potential landscape to one where the transitions occur between two states, the ones associated with the bottom of each well, while the dynamics is contained only in the transition rates. For all details on the general two-state approximation we refer to [9].

Up to first order in the amplitude $\delta \phi_{c}$ (assumed to be small in order to have a subthreshold periodic input) the transition rates $W_{i}$ adopt the form

$$
W_{i}=\tau_{0}^{-1} \exp \left\{-\frac{\Delta \mathcal{F}^{i}\left[\phi, k, y_{L}, t\right]}{\gamma}\right\}
$$

where

$$
\begin{aligned}
\Delta \mathcal{F}^{i}\left[\phi, k, y_{L}, t\right]= & \Delta \mathcal{F}^{i}\left[\phi, k, y_{L}\right] \\
& +\delta \phi_{c}\left(\frac{\partial \Delta \mathcal{F}^{i}\left[\phi, k, y_{L}\right]}{\partial \phi_{c}}\right)_{\phi_{c}=\phi_{c}^{*}} \cos (\omega t) .
\end{aligned}
$$

This yields for the transition probabilities

$$
W_{i} \simeq \frac{1}{2}\left(\mu_{i} \mp \alpha_{i} \frac{\delta \phi_{c}}{\gamma} \cos (\omega t)\right),
$$

where

$$
\begin{aligned}
\mu_{i} \approx \exp \left\{-\frac{\Delta \mathcal{F}^{i}\left[\phi, k, y_{L}\right]}{\gamma}\right\}, \\
\alpha_{i} \approx \pm \mu_{i}\left(\frac{d \Delta \mathcal{F}^{i}}{d \phi_{c}}\right)_{\phi_{c}^{*}}(i=1,2) .
\end{aligned}
$$

Using Eq. (3), it is clear that $\left.\left(d \Delta \mathcal{F}^{i} / d \phi_{c}\right)\right|_{\phi_{c}^{*}}$ can be obtained analytically.

These results allows us to calculate the autocorrelation function, the power spectrum density, and finally the SNR, which we indicate by $R$. The details of the calculation were shown in [9] and will not be repeated here. For $R$, and up to the relevant (second) order in the signal amplitude $\delta \phi_{c}$, we obtain [9]

$$
R=\frac{\pi}{4 \mu_{1} \mu_{2}} \frac{\left(\alpha_{2} \mu_{1}+\alpha_{1} \mu_{2}\right)^{2}}{\mu_{1}+\mu_{2}}=\frac{\pi}{4 \gamma^{2}} \frac{\mu_{1} \mu_{2}}{\mu_{1}+\mu_{2}} \Phi,
$$

where we have used the form of $\alpha_{i}$ to reduce the expression and

$$
\Phi=\left[\int_{-y_{L} / 2}^{y_{L} / 2} \phi_{h} \theta\left(\phi_{s t}(y)-\phi_{c}\right) d y\right]^{2}=\left[2 \phi_{h} y_{c}\left(y_{L}\right)\right]^{2} .
$$

We have now all the elements required to analyze the problem of SSSR.

Figure 3 shows the typical behavior of SR, but now-in the horizontal axis - the noise intensity is replaced by the the system length $y_{L}$, for fixed values of $k, \gamma$ (the noise intensity), and the ratio $\phi_{c} / \phi_{h}$. Such a response is the expected one for a system exhibiting SSSR. Within the context of NEP, it results clear that, in this kind of system, the phenomenon arises due to the breaking of the NEP's symmetry. This means that, as shown in Fig. 2, due to the variation of $y_{L}$, both attractors can exchange their relative stability. For a

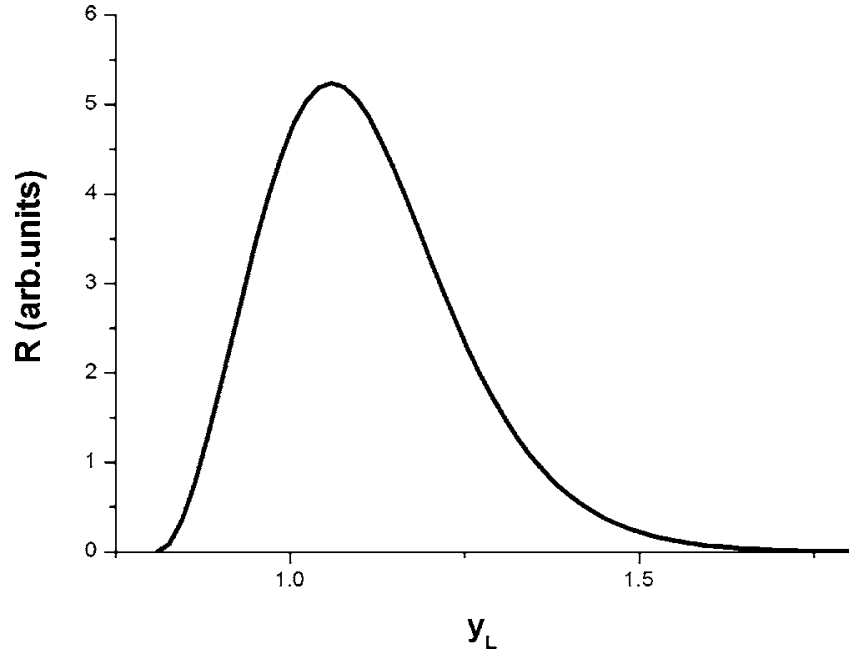

FIG. 3. SNR vs $y_{L}$ for $k=3, \gamma=0.1, D=1$, and $\phi_{c} / \phi_{h}=0.193$, showing the typical form of SNR when SSSR occurs.

value $y_{L}=y_{L}^{*} \approx 1$., both stable structures, the nonhomogeneous stable $\phi_{1}(y)$ and the trivial $\phi_{0}(y)$, have the same value for the NEP. When $y_{L}<y_{L}^{*}, \phi_{1}(y)$ becomes less stable than $\phi_{0}(y)$, making the transitions from $\phi_{1}(y)$ to $\phi_{0}(y)$ "easier" (the barrier is lower) than in the reverse direction, reducing the system's response. When $y_{L} \sim 0.72, \phi_{1}(y)$ and $\phi_{u}(y)$ coalesce and disappear, and the response is strictly zero (within the linear response implicit in the two-state approximation). When $y_{L}>y_{L}^{*}, \phi_{1}(y)$ becomes more stable than $\phi_{0}(y)$, making now the transitions from $\phi_{0}(y)$ to $\phi_{1}(y)$ easier than in the reverse direction, again reducing the system's response. Clearly, the system's response has a maximum when both attractors have the same stability $\left(y_{L}=y_{L}^{*}\right)$, and decays when departing from that situation. Hence, for this system and within this framework, SSSR arises as a particular case of the more general discussion done in [9].

A point worth to be remarked is the fact that, comparing Figs. 2(a) and 3, it is apparent that the value $y_{L}^{*}$, where the crossing between $\mathcal{F}^{1}$ and $\mathcal{F}^{0}$ occurs, is different from the value of $y_{L}$ at the maximum of the SNR curve. The origin of this difference is that, in principle, we have argued that the maximum of the SNR should be related to the potential being symmetrical (both wells having the same energy) [9]. However, the exact condition is that the transition rates between both wells should be equal. In general, due to the small differences between the curvatures at the bottom of both wells, those rates become equal for values of $y_{L}$ sightly different from the one in the symmetrical case. Here we have adopted a constant value for $\tau_{0}$, hence assuming equal curvatures. However, there is still a difference in the values of the $\alpha_{i}$, as $\left(d \Delta \mathcal{F}^{i} / d \phi_{c}\right)_{\phi_{c}^{*}}(i=1,2)$ are sightly different, a fact reflected in the dependence of $\Phi$ on $y_{L}$.

To conclude this section as well as for completeness, we consider the problem from a different angle. In Fig. 4 we show the curves of the SNR as a function of $k$, while keeping fixed values of $y_{L}$ and $\phi_{c} / \phi_{h}$. When $k$ is not too large, indicating a high degree of reflectiveness at the boundary (that is, a reduced exchange with the environment), we see that the SNR changes for $k$ varying from low to larger values, show- 


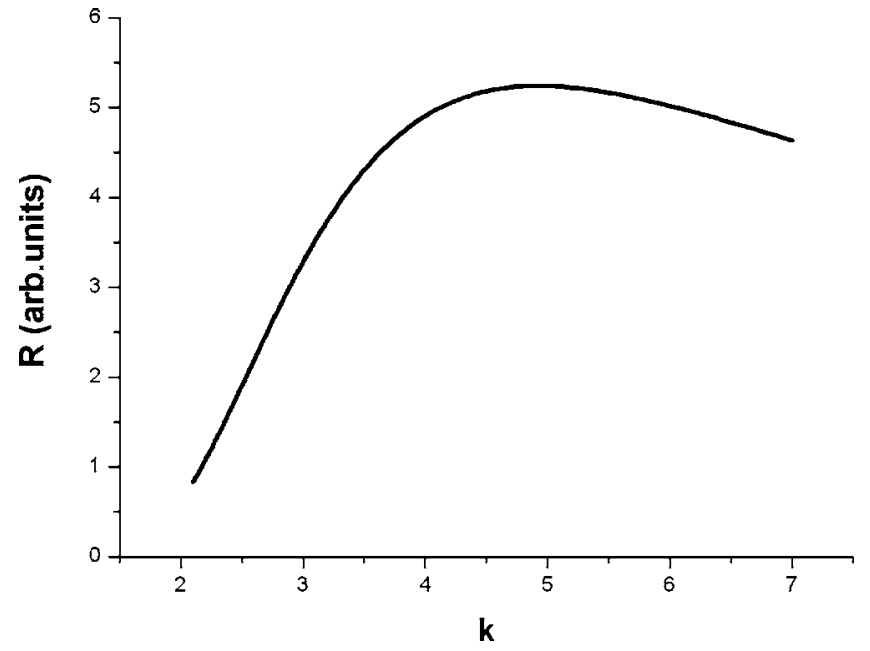

FIG. 4. SNR, vs $k$ for $y_{L}=1.2, \quad \gamma=0.1, D=1$, and $\phi_{c} / \phi_{h}$ $=0.193$. The robustness in the response is apparent.

ing a broad resonancelike curve. Remember that a large value of $k$ indicates that the system boundaries become absorbent. Such a broadening of the resonance indicates the robustness of the system's response when varying $k$, a parameter that somehow indicates a degree of coupling with the environment.

From the NEP's behavior we can put forward an argument to explain the present SNR result. From Fig. 2(b), we see on one hand that the difference between $\mathcal{F}^{1}$ and $\mathcal{F}^{u}$ has a strong variation with $k$, from very large values when $k$ is small to the asymptotic (Dirichlet-like) values reached for $k \rightarrow \infty$ (with $\mathcal{F}^{1}$ approaching a constant value smaller than $\mathcal{F}^{0}=0$ ); while the difference between $\mathcal{F}^{0}=0$ and $\mathcal{F}^{u}$ increases slowly with $k$ (until it reaches a positive constant value). Hence, as from Fig. 2(b) it is clear that in this situation the potential symmetry is always broken, we can understood the behavior of the SNR as follows: an initial increase of the SNR due to the reduction of the barrier when increasing $k$ from very small values, reaching a maximum for some intermediate value of $k$, and a final slow response decay due to the slow enlargement of the barrier when approaching the asymptotic Dirichlet values.

\section{THE GLOBAL COUPLING MODEL}

In this section we consider one of the models discussed in [20] from the point of view of the NEP approach. The model we refer to is described by a set of (globally) coupled nonlinear bistable oscillators,

$$
\begin{gathered}
\dot{x}_{j}=x_{j}-x_{j}^{3}+\frac{\varepsilon}{N} \sum_{k=1}^{N}\left(x_{k}-x_{j}\right)+\sqrt{2 \gamma} \xi_{j}(t)+f_{j}(t), \\
\dot{x}_{j}=-\frac{\partial}{\partial x_{j}} U(\{x\}, t)+\sqrt{2 \gamma} \xi_{j}(t),
\end{gathered}
$$

with $f_{j}(t)=A \cos (\omega t),\{x\}=\left(x_{1}, x_{2}, \ldots, x_{N}\right), \xi_{j}(t)$ are Gaussian noises with zero mean and $\left\langle\xi_{j}(t) \xi_{l}\left(t^{\prime}\right)\right\rangle=\delta_{j l} \delta\left(t-t^{\prime}\right)$, and where we have defined

$$
\begin{aligned}
U(\{x\}, t)= & U_{0}(\{x\})-A \cos (\omega t) \sum_{j=1}^{N} x_{j} \\
= & \sum_{j=1}^{N}\left(\frac{x_{j}^{4}}{4}-\frac{x_{j}^{2}}{2}\right)+\frac{\varepsilon}{2 N} \sum_{j=1}^{N} \sum_{k=1}^{N}\left(x_{k}-x_{j}\right)^{2} \\
& -A \cos (\omega t) \sum_{j=1}^{N} x_{j} \\
= & \sum_{j=1}^{N} u_{0}\left(x_{j}\right)+\frac{\varepsilon}{2 N} \sum_{j=1}^{N} \sum_{k=1}^{N}\left(x_{k}-x_{j}\right)^{2} \\
& -A \cos (\omega t) \sum_{j=1}^{N} x_{j} .
\end{aligned}
$$

Due to the structure of Eq. (11) it is clear that $U(\{x\})$, the potential function in Eqs. (11) and (12), is the discrete form of the NEP for this problem. For $A=0$ the stationary distribution of the multidimensional Fokker-Planck equation associated with Eq. (11) gives the result

$$
P_{\text {stat }}(\{x\}) \approx \exp \left(-\frac{U_{0}(\{x\})}{\gamma}\right)
$$

This potential has two attractors corresponding to $x_{1}=x_{2}$ $=\cdots=x_{N}= \pm 1$, and a barrier separating them at $x_{1}=x_{2}=\cdots$ $=x_{N}=0$.

Now, exploiting the same scheme as before but, as both attractors have the same energy, reduced to the symmetric case, we get for the SNR

$$
R \approx \exp \left(-\frac{\Delta U_{0}(\{x\})}{\gamma}\right) \approx \frac{N}{\gamma} \exp \left(-\frac{N \Delta u_{0}(X)}{\gamma}\right)
$$

where $X$ is a kind of "collective coordinate" (the one evolving along the trajectory joining both attractors), which passes though the saddle, and which can be approximately interpreted as $X \approx(1 / N) \sum_{j=1}^{N} x_{j}$. However, we need only evaluate the potential at the points $X=0, \pm 1$, and

$$
\Delta u_{0}(X)=u_{0}(X= \pm 1)-u_{0}(X=0) .
$$

Such a SNR clearly shows similar SSSR characteristics as those described in [20]. In this situation the NEP's symmetry is retained when varying $N$, while the wells are deepened (or the barrier separating them is enhanced). However, if we scaled out $N$, we find a constant effective potential $\left[u_{0}(X)\right]$, while the system shows an effective scaling of the noise with $N$. In this case we could speak of a noise scaled SSSR, in contrast to the previous case that could be called a NEP symmetry breaking SSSR.

In order to deepen our understanding of this case, let us analyze a continuous model, which is tightly connected with the previous discrete one. Consider a field $\psi(y, t)$, which behaves according to the following functional equation: 


$$
\begin{aligned}
\frac{\partial}{\partial t} \psi(y, t)= & \psi(y, t)-\psi(y, t)^{3}+\varepsilon \int_{\Omega}\left[\psi\left(y^{\prime}, t\right)-\psi(y, t)\right] d y^{\prime} \\
& +\xi(y, t)+f(t) \\
= & -\frac{\delta}{\delta \psi(y, t)} U(\psi(y, t))+\xi(y, t)
\end{aligned}
$$

with $f(t)=A \cos (\omega t)$, while for the noise we assume that, as before, it is white and Gaussian with $\langle\xi(y, t)\rangle=0$, and the correlation $\left\langle\xi(y, t) \xi\left(y^{\prime}, t^{\prime}\right)\right\rangle=2 \gamma \delta\left(y-y^{\prime}\right) \delta\left(t-t^{\prime}\right) . \Omega$ indicates the integration range, and $\delta / \delta \psi(y, t)$ is a functional derivative. We consider a finite system in the interval $y \in\left[-y_{L} / 2, y_{L} / 2\right]$, and assume Neumann boundary conditions. The form of the potential $U(\psi(y, t), t)$ becomes

$$
\begin{aligned}
U(\psi(y, t))= & U_{0}(\psi(y, t))-F(\psi(y, t)) \\
= & \int_{\Omega} d y u_{0}(\psi(y, t))+\frac{\varepsilon}{2} \int_{\Omega} d y \\
& \times \int_{\Omega} d y^{\prime}\left[\psi\left(y^{\prime}, t\right)-\psi(y, t)\right]^{2}-F(\psi(y, t)),
\end{aligned}
$$

where

$$
u_{0}(\psi(y, t))=\left(\frac{\psi(y, t)^{4}}{4}-\frac{\psi(y, t)^{2}}{2}\right)
$$

and

$$
F(\psi(y, t))=A \cos (\omega t) \int_{\Omega} \psi(y, t) d y .
$$

This potential is clearly similar to the one discussed in [8], but with the local (diffusive) coupling being zero, and the nonlocal contribution becoming "global." For $A=0$ the stationary distribution of the multidimensional Fokker-Planck equation associated with Eq. (15) gives the result

$$
P_{\text {stat }}\left(\psi_{\text {stat }}(y)\right) \approx \exp \left(-\frac{U_{0}\left(\psi_{\text {stat }}(y)\right)}{\gamma}\right),
$$

with $\gamma$ the noise intensity. This potential has two attractors corresponding to the constant fields $\psi_{\text {stat }}(y)= \pm 1$, and a barrier separating them at $\psi_{\text {stat }}(y)=0$.

Hence, exploiting the same scheme as in the previous section, but reduced to the symmetric case as both attractors have the same energy, we get for the SNR

$$
R \approx \exp \left(-\frac{\Delta U_{0}\left(\psi_{\text {stat }}(y)\right)}{\gamma}\right) \approx \frac{y_{L}}{\gamma} \exp \left(-\frac{y_{L} \Delta u_{0}\left(\psi_{\text {stat }}(y)\right)}{\gamma}\right) .
$$

where

$$
\Delta u_{0}\left(\psi_{\text {stat }}(y)\right)=u_{0}\left(\psi_{\text {stat }}(y)= \pm 1\right)-u_{0}\left(\psi_{\text {stat }}(y)=0\right) .
$$

This SNR clearly shows the same SSSR characteristics as those described for the discrete case, where the role of $N$ (number of elements) is now played by $y_{L}$ (size of the system).
Note that Eq. (15) corresponds to the continuous limit of Eq. (11) and the result for the discrete case [Eq. (14)] is recovered in Eq. (18) for the normalized noise intensity $\gamma_{d c}$ $=\gamma / \Delta x$ (with $\gamma_{d c}$ the noise intensity for the discrete case), and $y_{L}=N \Delta x$.

To conclude this section, we refer to another case discussed in [20], the one corresponding to the Ising model. Such a case has many similarities with the case of the set of coupled nonlinear bistable oscillators discussed above. It can be described in a similar way to the case above. That means we could also find an effective potential playing the role of the NEP, having two attractors (corresponding to all spins up or all down), a barrier corresponding to a mixed state, whose height depends linearly on $N$ (the number of spins). The final result will be similar to the one in Eq. (14) above.

\section{MULTIPLICATIVE NOISE CASE}

\section{A. Brief review of the model}

The basic model to be considered in this section is the same one studied in [16], and consist of the following ensemble of nonlinear coupled oscillators, described in terms of a continuous field:

$$
\frac{\partial}{\partial t} \phi(y, t)=\frac{\partial}{\partial y}\left(D(\phi) \frac{\partial}{\partial y} \phi\right)+f(\phi)+\frac{1}{\sqrt{D(\phi)}} \xi(y, t) .
$$

Here $\xi(y, t)$ is again a Gaussian white noise with zero mean and correlation $\left\langle\xi(y, t) \xi\left(y^{\prime}, t^{\prime}\right)\right\rangle=2 \gamma \delta\left(y-y^{\prime}\right) \delta\left(t-t^{\prime}\right), \gamma$ being the noise intensity. $D(\phi)$ is a field-dependent diffusivity and the coefficient of the noise term guarantee that a fluctuationdissipation relation is satisfied [28]. The nonlinearity $f(\phi)$ which drives the dynamics in absence of noise is chosen to be monostable, and we adopt a density-dependent diffusion coefficient to generate a noise-induced bistable dynamics. In particular, as in [16], we use

$$
D(\phi)=\frac{D_{0}}{1+h \phi^{2}}, \quad f(\phi)=-\phi^{3}+b \phi^{2},
$$

$D_{0}, h$, and $b$ being positive constants. We will consider a finite system, limited to $\left(-y_{L} / 2 \leqslant y \leqslant y_{L} / 2\right)$, and assume Dirichlet boundary conditions $\phi\left( \pm y_{L} / 2, t\right)=0$.

As we are considering the Stratonovich interpretation, the stationary solution of the probability $P_{s t}(\phi)$ of the stochastic field $\phi(x, t)$ given by Eq. (19) can be written [29] in terms of an effective potential

$$
P_{s t}(\phi) \sim \exp \left(-V_{e f f}[\phi] / \gamma\right)
$$

with

$$
V_{e f f}[\phi]=\int_{-y_{L} / 2}^{y_{L} / 2} d y\left\{\frac{1}{2}\left(D(\phi) \frac{\partial}{\partial y} \phi\right)^{2}-U(\phi)-\lambda \ln D(\phi)\right\},
$$

where $U(\phi)=\int_{0}^{\phi} D\left(\phi^{\prime}\right) f\left(\phi^{\prime}\right) d \phi^{\prime}$. Here $\lambda$ is a renormalized parameter, related to $\gamma$ by $\lambda=(\gamma / 2 \Delta y)$ in a square discrete lattice, where $\Delta y$ is the lattice parameter [29]. The extremes of $V_{\text {eff }}$ (stationary noise-induced structures of the effective 
dynamics) can be computed from an equation whose form and the one of the effective force $F_{\text {eff }}(\phi)$ are given by Eqs. (9) and (13) in [16]. The coefficients in those equations depend on the system's parameters, in particular on the renormalized noise intensity $\lambda$. There is a trivial homogeneous structure $\phi=0$ and two nonhomogeneous patterns, an unstable (saddle) $\phi_{u}$ and a stable one $\phi_{s}$ (see Ref. [16]).

We note that in the deterministic problem we have a monostable [30] reaction term $(\lambda=0)$. As we increase the noise intensity, due to the noise effects, we have an effective nonlinear term $F_{\text {eff }}$ that becomes bistable [in the interval 0 $\left.<\lambda<D_{0} /(2 h)\right]$ and finally monostable for $\lambda>D_{0} /(2 h)$ (reentrance effect). We also remark that $\phi=0$ is always a root of $F_{e f f}$, and it is an extremum of $V_{e f f}[\phi]$ for all values of $\lambda$. In what follows we will call this structure $\phi_{0}$. The typical form of the nonlinearity $\left(F_{\text {eff }}\right)$ for various values of $\lambda$ has been shown in Fig. 1 of Ref. [16]. As a final remark, the situation here is that the same noise that induces the patterns and the bistability is the one inducing the transitions among them and the SR phenomenon.

\section{B. System size stochastic resonance}

To analytically describe the stochastic resonance, we resort again to the two-state approach in the adiabatic limit [1]. As indicated before, all details about the procedure and the evaluation of the SNR can be found in [9]. The system is now subject to a time periodic subthreshold signal $b=b_{0}$ $+S(t)$ where $S(t)=\Delta b \sin \left(\omega_{0} t\right)$. Up to first order in the small amplitude $\Delta b$ the transition rates $W_{i}$ take the form

$$
W_{i}(t)=\mu_{i} \mp \alpha_{i} \Delta b \sin \left(\omega_{0} t\right),
$$

where the constants $\mu_{1,2}$ and $\alpha_{1,2}$ are obtained from the Kramers-like formula for the transition rates [27]

$$
W_{\phi_{i} \rightarrow \phi_{j}}=\frac{\beta_{+}}{2 \pi}\left[\frac{\operatorname{det} V_{e f f}\left(\phi_{i}\right)}{\left|\operatorname{det} V_{e f f}\left(\phi_{s}\right)\right|}\right]^{1 / 2} \exp \left(\frac{V_{e f f}\left(\phi_{i}\right)-V_{e f f}\left(\phi_{s}\right)}{\gamma}\right) .
$$

Here $\beta_{+}$is the unstable eigenvalue of the deterministic flux at the relevant saddle point $\left(\phi_{s}\right)$ and

$$
\mu_{1,2}=W_{1,2 S(t)=0}, \quad \alpha_{1,2}=\left.\mp \frac{d W_{1,2}}{d S(t)}\right|_{S(t)=0} .
$$

We note in passing that, due to the system's sensitivity to small variations in the parameters, and at variance with the case studied in Sec. II, here we require the evaluation of the prefactor in $W_{\phi_{i} \rightarrow \phi_{j}}$.

As before, these results allows us to calculate the autocorrelation function, the power spectrum, and finally the SNR (indicated by $R$ ). For $R$, up to the relevant (second) order in the signal amplitude $\Delta b$, similarly to Eqs. (9) and (10), we obtain

$$
R=\frac{\pi}{4 \gamma^{2}} \frac{\mu_{1} \mu_{2}}{\mu_{1}+\mu_{2}} \Phi
$$

where now

$$
\Phi=\int_{-y_{L} / 2}^{y_{L} / 2} d y \int_{\phi_{0}}^{\phi_{s}(y)} D\left(\phi^{\prime}\right) \phi^{\prime 2} d \phi^{\prime}
$$

gives a simultaneous measure of the spatial coupling [through $D(\phi)$ ] and the system size extension (through $\int d y$ ). In our previous work [16] we have found that the dependence of the SNR as a function of $\lambda$ is maximum at the symmetric situation $\lambda=\lambda_{c}=0.8$, where both stable structures $\left(\phi_{0}\right.$ and $\left.\phi_{s}\right)$ have the same stability $\left(V_{\text {eff }}\left[\phi_{0}\right]=V_{\text {eff }}\left[\phi_{s}\right]\right)$.

To analyze the system size dependence of the SR, we fix the renormalized noise intensity $\left(\lambda=\lambda_{c}=0.8\right)$ and the parameters $D_{0}=1, b=2$, and $h=1 / 2$; and only change the length $y_{L}$ (with fixed lattice parameter $\Delta x$ ).

It is worth remarking here that in [20] what is varied is the length of the lattice, while the noise intensity, the coupling, etc., are kept constant. In the present case, due to the characteristics of the model, we have that as $y_{L}$ is varied, the fields change inducing the change of the diffusive coupling, making a strong difference from the case in [20].

At this point we can just analyze the dependence of the SNR with $y_{L}$ as was done in Sec. II. However, as was indicated near the end of that section, we use an alternative form of analysis, looking at the scaling of the potential with $y_{L}$. We consider the following transformations:

$$
\begin{gathered}
y \rightarrow x=\frac{y}{y_{L}}, \\
D_{o} \rightarrow D_{1}=\frac{D_{o}}{y_{L}}, \\
D(\phi)=\frac{D_{o}}{1+h \phi^{2}} \rightarrow D_{1}(\phi)=\frac{D_{1}}{1+h \phi^{2}} ;
\end{gathered}
$$

the effective potential can be written as

$$
\begin{aligned}
V_{\text {eff }}[\phi]= & y_{L} \int_{-1 / 2}^{1 / 2} d x\left\{-U(\phi)+\frac{1}{2}\left[\frac{D(\phi)}{y_{L}} \frac{\partial}{\partial x} \phi\right]^{2}\right\} \\
& -\lambda y_{L} \int_{-1 / 2}^{1 / 2} d x \ln D(\phi),
\end{aligned}
$$

which finally yields [using the previous definition $D(\phi) / y_{L}$ $\left.=D_{1}(\phi)\right]$

$$
\begin{aligned}
V_{\text {scal }}\left(y_{L}\right)= & V_{e f f}[\phi] / y_{L} \\
= & \int_{-1 / 2}^{1 / 2} d x\left\{-U(\phi)+\frac{1}{2}\left[D_{1}(\phi) \frac{\partial}{\partial x} \phi\right]^{2}\right. \\
& \left.-\lambda \ln D_{1}(\phi)\right\}-\lambda \ln y_{L} .
\end{aligned}
$$

Here it becomes apparent that this scaling yields a logarithmic length contribution to the scaled potential.

The stationary solution $P_{s t}(\phi)$ of the stochastic field $\phi(y, t)$ can be written

$$
P_{s t}(\phi) \sim \exp \left[-V_{s c a l}\left(y_{L}\right) / \gamma_{x}\left(y_{L}\right)\right],
$$

with $\gamma_{x}\left(y_{L}\right)=\gamma / y_{L}$. 


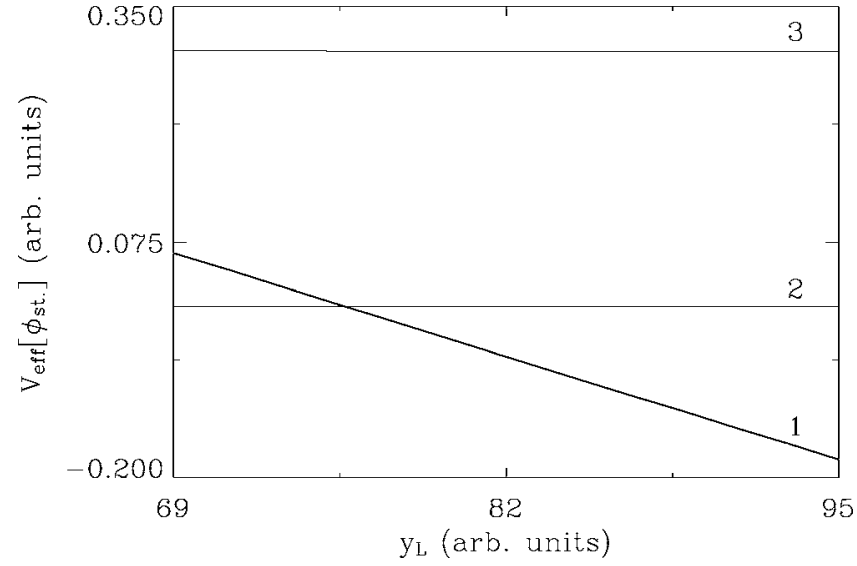

FIG. 5. Nonequilibrium potential $V_{e f f}\left[\phi_{s t}\right]$ evaluated in the stationary structures as a function of the system size $y_{L}$. Curves correspond to (1) stable $\left(\phi_{s}\right),(2)$ homogeneous $\left(\phi_{0}\right)$, and (3) unstable $\left(\phi_{u}\right)$ patterns. Note the global stabilization of the nontrivial stable pattern for high values of $y_{L}$.

We can also consider the scaling of the spatial factor $\Phi$ [see Eq. (22)]. The result is

$$
\begin{aligned}
\Phi & =y_{L} \int_{-1 / 2}^{1 / 2} d x \int_{\phi_{0}}^{\phi_{s}(x)} D\left(\phi^{\prime}\right) \phi^{\prime 2} d \phi \\
& =y_{L} \frac{D_{0}}{h} \int_{-1 / 2}^{1 / 2} d x\left\{\phi_{s}(x)-\frac{\arctan \left[\sqrt{h} \phi_{s}(x)\right]}{\sqrt{h}}\right\} .
\end{aligned}
$$

Hence, we have the dependence of the NEP as well as the transition rates [Eq. (20)] and finally of the SNR [Eq. (21)], on the system length (or the number of coupled elements for discrete systems). To illustrate this, in Fig. 5 we show $V\left(\phi_{e f f}\right)$ as a function of $y_{L}$. The behavior shown in this figure is analogous to the one observed in Fig. 1(a). Hence, we can anticipate the existence of SSSR in this system.

We see that for small $y_{L}$, small size effects increase the NEP values of the nonhomogeneous structures, and the uniform state results to be the most stable one. For $y_{L}=y_{L_{c}}$ both the NEPs for $\phi_{0}$ and $\phi_{1}$ become equal with a null value. For $y_{L}>y_{L_{c}}$, the NEP of the uniform state becomes metastable, and the nonuniform pattern is the globally stable attractor. The transition rates also reflect this fact (they are decreasing functions of $y_{L}$ ). However, we expect that $\Phi$ (which depends on the system size) increases with $y_{L}$, and due to the interplay between the rates and the behavior of $\Phi$, a SSSR behavior could be expected. Such a behavior becomes apparent in Fig. 6.

We can make the following interpretation in terms of $V_{\text {scal }}\left(y_{L}\right)$, the effective NEP [or scaled form of the NEP, Eq. (24)], and the the scaling of the noise intensity: in Fig. 7 we depict the form of $V_{\text {scal }}\left(y_{L}\right)$ as a function of $y_{L}$. It is clear that, even though it is weak, the dependence of $V_{\text {scal }}\left(y_{L}\right)$ on $y_{L}$ still shows the change in the relative stability of the attractors, while we have the scaling of the noise intensity with $y_{L}$. Hence, we can argue that there is a kind of effective entanglement between the symmetry breaking and the noise scaling.

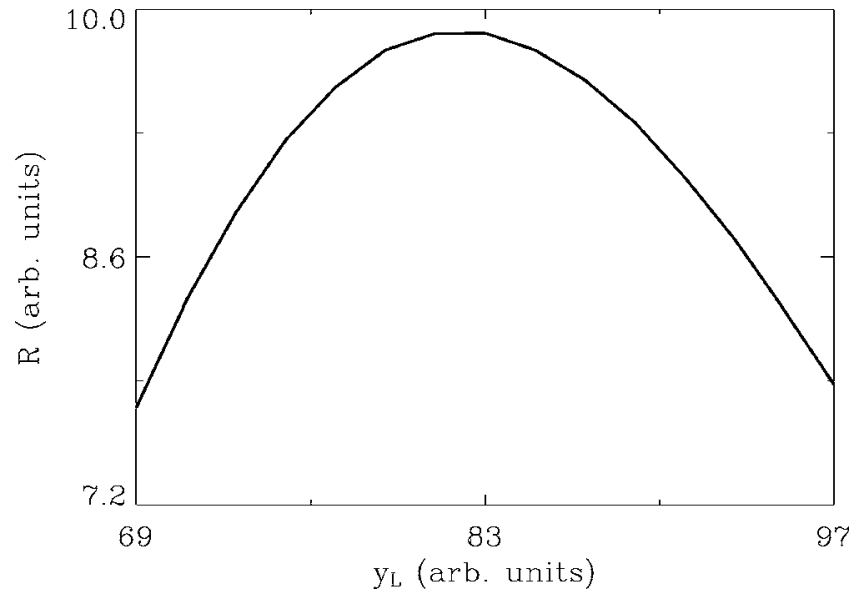

FIG. 6. SNR vs $y_{L}$. As indicated in the text, we fixed $D_{o}=1, h$ $=0.5, b=2$, and $\lambda=\lambda_{c}=0.8$. The behavior of $R$ with $y_{L}$ is the typical one for SSSR.

There is one point worth remarking. From Figs. 5 and 6, it is apparent that the value of $y_{L}$ at which the crossing between $V_{e f f}\left[\phi_{s}\right]$ and $V_{e f f}\left[\phi_{0}\right]$ occurs $\left(y_{L}^{*} \sim 76\right)$, and the one where $R$ has the maximum $\left(y_{L}^{\dagger} \sim 82\right)$, do not coincide. The explanation of this fact is the same one introduced near the end of Sec. II. Here we find differences in both the values of $\mu_{i}$ and $\alpha_{i}$; the latter are reflected in the dependence of $\Phi$ on $y_{L}$.

\section{CONCLUSIONS}

The study of SR in extended or coupled systems, motivated by both some experimental results and the growing technological interest, has recently attracted considerable attention [5-10,13]. In this paper we have discussed a unified way to analyze the SSSR phenomenon by focusing on the scaling properties with $y_{L}$ of two relevant magnitudes that control the process: the NEP and the noise, respectively. In particular we have discussed in detail two of the cases analyzed in [22] and presented a third one that corresponds to

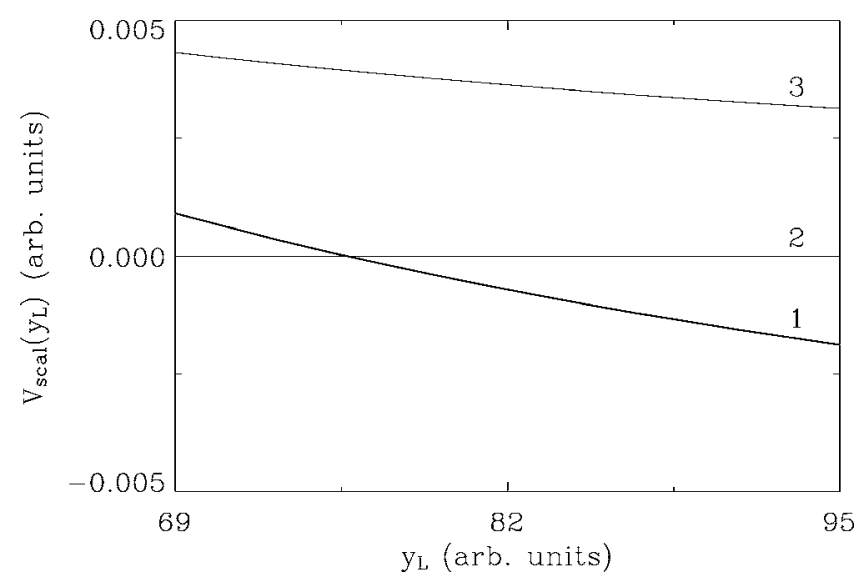

FIG. 7. $V_{\text {scal }}\left(y_{L}\right)=V_{\text {eff }}\left(y_{L}\right) / y_{L}$ evaluated on the stationary structures as a function of the system size $y_{L}$. Curves correspond to (1) stable $\left(\phi_{s}\right),(2)$ homogeneous $\left(\phi_{0}\right)$, and (3) unstable $\left(\phi_{u}\right)$ patterns. These correspond, to the same curves as in Fig. 5. 
the study of the system size dependence in the same system studied in [16].

In the first case we focused on a simple reaction-diffusion model with a known form of the NEP [23,24], and-as in all three cases-considering the adiabatic limit and exploiting the two-state approximation, we were able to clearly quantify the system size dependence of the SNR. We have shown that in this case, SSSR is associated with a NEP's symmetry breaking. For the second case we analyzed the model of globally coupled nonlinear oscillators discussed in [20], and have shown that it can also be described within the NEP framework, but now SSSR arises through an effective scaling of the noise intensity with the system size. For the third studied case, we have obtained the exact form of the noiseinduced patterns (both the stable and unstable ones) as well as the analytical expression of the NEP. The interplay of the transition rates, which are essentially decreasing functions of $y_{L}$, and the behavior of $\Phi$, which increases with $y_{L}$, explains the existence of a maximum in the SNR for a specific length of the system and a fixed noise intensity. What arose here, through an alternative form of analysis, is that there is a kind of entrainment between the symmetry breaking of the NEP as described in [22], together with a scaling of noise intensity with the system size as in [20].

The results found in this work clearly show that the nonequilibrium potential (even if not known in detail; see, for instance, [31]) offers a very useful framework to analyze a wide spectrum of characteristics associated with SR in spatially extended or coupled systems. Within this framework the phenomenon of SSSR looks, like other aspects of SR in extended systems [9], to be a natural consequence of a breaking of the symmetry of the NEP or an effective scaling of the noise intensity as in [20], or possibly as a mixture between the two aspects.
In addition, in the first studied case, we have seen a form of resonant behavior through the variation of the coupling with the surroundings. In such a case the system's response to an external signal becomes more robust, that is, less sensitive to the precise value of the albedo parameter. This fact opens further possibilities for analyzing and interpreting the behavior of some biological systems [32].

As a final comment, the main difference between the first and third cases when compared with the second one is that in the two former cases we have local interactions with some BCs (albedo or Dirichlet BCs), while the latter has a nonlocal coupling together with boundary conditions that could be assumed as Neumann. From our results, it is possible to argue that the effective scaling of the noise that arises in the second case comes from the nonlocal interaction, and not from the BCs. To make this aspect more obvious, we plan to study, within the present framework, the competition between local and nonlocal spatial couplings $[8,10]$ that arises in some multicomponent models. Also, the consideration of more general systems with several components will allow us to analyze the system size dependence of SR between patterns in general activator-inhibitor-like systems. All these aspects will be the subject of further work.

\section{ACKNOWLEDGMENTS}

The authors thanks R. Toral, C. Tessone, A. D. Sánchez, and S. Mangioni for fruitful discussions. H.S.W. acknowledges partial support from ANPCyT, Argentine, and thanks the European Commission for support at the Universidad de Cantabria, Spain. G.G.I. thanks the Departamento de Fisica Interdisciplinar, IMEDEA, UIB, Spain, for the kind hospitality extended to him.
[1] L. Gammaitoni, P. Hänggi, P. Jung, and F. Marchesoni, Rev. Mod. Phys. 70, 223 (1998).

[2] T. Wellens, V. Shatokin, and A. Buchleitner, Rep. Prog. Phys. 67, 45 (2004).

[3] J. K. Douglas et al., Nature (London) 365, 337 (1993); J. J. Collins et al., ibid. 376, 236 (1995); S. M. Bezrukov and I. Vodyanoy, ibid. 378, 362 (1995).

[4] A. Guderian, G. Dechert, K. Zeyer, and F. Schneider, J. Phys. Chem. 100, 4437 (1996); A. Förster, M. Merget, and F. Schneider, ibid. 100, 4442 (1996); W. Hohmann, J. Müller, and F. W. Schneider, ibid. 100, 5388 (1996).

[5] J. F. Lindner, B. K. Meadows, W. L. Ditto, M. E. Inchiosa, and A. Bulsara, Phys. Rev. E 53, 2081 (1996).

[6] A. Bulsara and G. Schmera, Phys. Rev. E 47, 3734 (1993); P. Jung, U. Behn, E. Pantazelou, and F. Moss, Phys. Rev. A 46, R1709 (1992); P. Jung and G. Mayer-Kress, Phys. Rev. Lett. 74, 2130 (1995); J. F. Lindner, B. K. Meadows, W. L. Ditto, M. E. Inchiosa, and A. Bulsara, ibid. 75, 3 (1995); F. Marchesoni, L. Gammaitoni, and A. Bulsara, ibid. 76, 2609 (1996).

[7] H. S. Wio, Phys. Rev. E 54, R3075 (1996); H. S. Wio and F. Castelpoggi, in Proceedings of the Conference UPoN'96, ed- ited by C. R. Doering, L. B. Kiss, and M. Schlesinger (World Scientific, Singapore, 1996); F. Castelpoggi and H. S. Wio, Europhys. Lett. 38, 91 (1997).

[8] F. Castelpoggi and H. S. Wio, Phys. Rev. E 57, 5112 (1998).

[9] S. Bouzat and H. S. Wio, Phys. Rev. E 59, 5142 (1999).

[10] H. S. Wio, S. Bouzat, and B. von Haeften, in Proceedings of the 21st IUPAP International Conference on Statistical Physics, STATPHYS21, edited by A. Robledo and M. Barbosa [Physica A 306, 140 (2002)].

[11] C. J. Tessone, H. S. Wio, and P. Hänggi, Phys. Rev. E 62, 4623 (2000).

[12] M. A. Fuentes, R. Toral, and H. S. Wio, Physica A 295, 114 (2001).

[13] B. von Haeften, R. Deza, and H. S. Wio, Phys. Rev. Lett. 84, 404 (2000).

[14] R. Graham and T. Tel, in Instabilities and Non-equilibrium Structures III, edited by E. Tirapegui and W. Zeller (Kluwer, Dordrecht, 1991); H. S. Wio, in Fourth Granada Seminar in Computational Physics, edited by P. Garrido and J. Marro (Springer-Verlag, Berlin, 1997), p. 135.

[15] G. Izús et al., Phys. Rev. E 52, 129 (1995); G. Izús et al., Int. J. Mod. Phys. B 10, 1273 (1996); D. H. Zanette, H. S. Wio, 
and R. Deza, Phys. Rev. E 53, 353 (1996); F. Castelpoggi, H. S. Wio, and D. H. Zanette, Int. J. Mod. Phys. B 11, 1717 (1997).

[16] B. von Haeften et al., Phys. Rev. E 69, 021107 (2004); in Noise in Complex Systems and Stochastic Dynamics, edited by Z. Gingl, J. M. Sancho, L. Schimansky-Geier, and J. Kerstez, Proceedings of SPIE Vol. 5471, (SPIE, Bellinghan, WA, 2004), pp. 258-265.

[17] G. Schmid, I. Goychuk, and P. Hänggi, Europhys. Lett. 56, 22 (2001); Phys. Biol. 1, 61 (2004).

[18] P. Jung and J. W. Shuai, Europhys. Lett. 56, 29 (2001); J. W. Shuai and P. Jung, Phys. Rev. Lett. 88, 068102 (2003).

[19] R. Toral, C. Mirasso, and J. Gunton, Europhys. Lett. 61, 162 (2003).

[20] A. Pikovsky, A. Zaikin, M. A. de la Casa, Phys. Rev. Lett. 88, 050601 (2002).

[21] C. J. Tessone, R. Toral, Physica A 351, 106 (2005).

[22] H. S. Wio, e-print cond-mat/0410464, in Proceedings of the 18th International Conference on Noise and Fluctuations, AIP Conf. Proc. (AIP, Melville, NY, in press).

[23] H. S. Wio, An Introduction to Stochastic Processes and Nonequilibrium Statistical Physics (World Scientific, Singapore, 1994), Chap. 5; A. S. Mikhailov, Foundations of Synergetics I
(Springer-Verlag, Berlin, 1990).

[24] W. J. Skocpol, M. R. Beasley, and M. Tinkham, J. Appl. Phys. 45, 4054 (1974); B. Ross and J. D. Litster, Phys. Rev. A 15, 1246 (1977); D. Bedeaux and P. Mazur, Physica A 105, 1 (1981).

[25] C. L. Schat and H. S. Wio, Physica A 180, 295 (1992).

[26] J. García-Ojalvo and J. M. Sancho, Noise in Spatially Extended Systems (Springer-Verlag, New York, 1999).

[27] P. Hänggi, P. Talkner, and M. Borkovec, Rev. Mod. Phys. 62, 251 (1990).

[28] K. Kitahara and M. Imada, Suppl. Prog. Theor. Phys. 64, 65 (1978).

[29] M. Ibañes, J. García-Ojalvo, R. Toral, and J. M. Sancho, Phys. Rev. Lett. 87, 020601 (2001).

[30] Monostable means here that the function derives from a monostable potential.

[31] H. S. Wio, M. N. Kuperman, F. Castelpoggi, G. Izús, and R. Deza, Physica A 257, 275 (1998).

[32] A. Fulinski, Z. Grzywna, I. R. Mellor, Z. Siwy, and P. N. R. Usherwood, Phys. Rev. E 58, 919 (1998); Sz. Mercik and K. Weron, ibid. 63, 051910 (2001); I. Goychuk and P. Hanggi, ibid. 69, 021104 (2004). 